\title{
Bipolar Conductance Switching of Single Anthradithiophene Molecules
}

\author{
Bogdana Borca, ${ }^{*, \dagger, \perp}$ Verena Schendel, ${ }^{\dagger, \perp}$ Rémi Pétuya, ${ }^{\ddagger}$ Ivan Pentegov, ${ }^{\dagger}$ \\ Tomasz Michnowicz, ${ }^{\dagger}$ Ulrike Kraft, ${ }^{\dagger}$ Hagen Klauk, ${ }^{\dagger}$ Andrés Arnau,,$\uparrow$ Peter \\ Wahl, ${ }^{\dagger, \S}$ Uta Schlickum, ${ }^{* \dagger \dagger}$ and Klaus Kern ${ }^{\dagger, \|}$ \\ $\dagger$ †ax-Planck-Institute for Solid State Research, 70569 Stuttgart, Germany \\ $\ddagger$ Donostia International Physics Centre, E-20018 Donostia - San Sebastián, Spain \\ IDepartamento de Fisica de Materiales UPV/EHU and CFM Donostia, Centro Mixto \\ CSIC-UPV/EHU, E-20018 Donostia - San Sebastián, Spain \\ $\S S U P A$, School of Physics and Astronomy, University of St Andrews, North Haugh, St \\ Andrews, KY16 9SS, United Kingdom \\ \|Institut de Physique de la Matière Condensée, École Polytechnique Fédérale de Lausanne \\ (EPFL), CH-1015 Lausanne, Switzerland \\ $\perp$ Equal contribution \\ E-mail: b.borca@fkf.mpg.de; u.schlickum@fkf.mpg.de \\ Phone: +49 (0)711 689-1539. Fax: +49 (0)711 689-1662
}

\begin{abstract}
Single molecular switches are basic device elements in organic electronics. The pentacene analog anthradithiophene (ADT) shows a fully reversible binary switching between different adsorption conformations on a metallic surface induced by a charge transfer. These transitions are activated locally in single molecules in a low temperature Scanning Tunneling Microscope (STM). The switching induces changes between
\end{abstract}


bistable orbital structures and energy level alignment at the interface. The most stable geometry, the "off" state, which all molecules adopt upon evaporation, corresponds to a short adsorption distance at which the electronic interactions of the acene rings bend the central part of the molecule towards the surface accompanied by a significant charge transfer from the metallic surface to the ADT molecules. This leads to a shift of the lowest unoccupied molecular orbital (LUMO) down to the Fermi level $\left(E_{F}\right)$. In the "on" state the molecule has a flat geometry at a larger distance from the surface, consequently the interaction is weaker, resulting in a negligible charge transfer with an orbital structure resembling the highest occupied molecular orbital (HOMO) when imaged close to the $E_{F}$. The potential barrier between these two states can be overcome reversibly by injecting charge carriers locally into individual molecules. Voltage controlled current traces show a hysteresis characteristic of a bipolar switching behavior. The interpretation is supported by first principles calculations.

\section{Keywords}

cis/trans ADT isomers, $\mathrm{Cu}(111)$, STM, DFT, conformational and electronic switches

A visionary perspective of molecular electronics is the use of single molecules as functional entities in electronic devices. ${ }^{1}$ In this context, important components are molecular switches, ${ }^{2,3}$ which can be interconverted reversibly between two or more stable states. Scanning Tunneling Microscopy and Spectroscopy (STM/STS) represents an ideal tool to characterize and manipulate single atoms and molecules on surfaces. ${ }^{4}$ Several switching mechanisms have been explored previously with molecular and submolecular resolution and have been induced locally by tunneling electrons, ${ }^{5-23}$ by an applied electric field ${ }^{24,25}$ or by combined stimuli $^{26-28}$ in the STM tunneling junction. Changes of the molecular configurations were identified as changes of the folding in complex organic molecules, ${ }^{5}$ different types of isomerization processes, ${ }^{7,13,15,18-24,28}$ changes of the atomic position in metal-organic complexes, ${ }^{6,26}$ 
formation and dissociation of chemical bonds. ${ }^{8-11,16,17,25}$ These conformational changes may be accompanied by charging and discharging processes. ${ }^{12-16,27,28}$ Recently, another scenario was proposed theoretically in which a switching process of an individual molecule is related to bistable electronic states. These are associated with different bonding strengths and hybridization degrees of the molecules to a metallic substrate, resulting in a change of the bending of the molecular conformation concomitant with a change of the adsorption distance. ${ }^{29}$

In this study, we focus on single molecular switches consisting of individual anthradithiophene (ADT) molecules. ADT is a structural analog of pentacene, with two sulfur containing groups at each molecular terminal side representing cis and trans diastereomers. ${ }^{30}$ The switching of individual molecules is activated locally by injecting charge carriers from the STM tip apex into the molecule. The molecules are reversibly interconverted by bipolar voltage pulses between two different adsorption geometries that correspond to two different electronic conformations of the molecule. The characteristic parameter responsible for the structural and electronic changes is the adsorption distance that determines the bonding strength and the degree of hybridization between the ADT molecule and the surface, similar to the recently proposed case of benzene derivatives. ${ }^{29}$ Our first-principles calculations using van der Waals density functionals where exchange and correlation are treated consistently (vdW-DF-cx), ${ }^{31,32}$ allow us to propose a model based on the appearance of two adsorption states corresponding to two different geometric (bent and planar) and electronic conformations. These two conformations are induced by two main driving forces, the S-Cu bond length and the adsorption constraint for an optimal binding of the acene groups to the substrate, similar to pentacene. ${ }^{33,34}$ 


\section{Results and discussion}

Individual ADT molecules deposited at $200 \mathrm{~K}$ on the $\mathrm{Cu}(111)$ surface, adsorb with their long axis along the high symmetry directions of the substrate, where cis and trans isomers adopt the same adsorption conformation (Figure 1 a). In topographic images acquired with a metallic tip the two isomers are practically indistinguishable and all molecules appear in a "dumbbell-like" shape. Functionalizing the STM tip with a single ADT molecule (by picking up the encircled molecule in Figure 1 a) the orbital structure can be resolved (Figure 1 b) and thus the cis and trans diastereomers can be identified. Different configuration characteristic for each isomer at the molecular extremities, with higher charge density on the same side (cis) or to the opposite side (trans) are observed, as indicated by the arrows in Figure 1 $(\mathrm{c}, \mathrm{d})$.

Placing the tip above the center of the molecules, as shown in Figure 2 (a, b), a negative voltage pulse of $-0.5 \mathrm{~V}$ was applied. During this measurement the feedback loop was opened and the tunneling current recorded. After a certain time, an abrupt change in the tunneling current, i.e., a step-like (for cis-ADT) and a double step-like (for trans-ADT) behavior is observed (Figure $2 \mathrm{c}, \mathrm{d}$ ). Rescanning the same molecules reveals that the increase in the current is associated with a change in the topographic appearance (Figure $2 \mathrm{a}, \mathrm{b}$ ). In addition, in the switched conformation the trans-ADT molecule is rotated by about $10^{\circ}$ (Figure 2 b), which can be related to the double step feature in the current trace (Figure 2 d). Switching the molecule back to the initial state is induced by a positive voltage (Figure $2 \mathrm{c}, \mathrm{d}$ lower panel). In the following, we will refer to the initial adsorption conformation as the "off" state, and the conformation related to a higher conductance (in open feedback-loop conditions) as the "on" state.

The measurements suggest that the two different adsorption conformations of ADT molecules rely on the interplay between the $\mathrm{S}$ containing groups and the acene group position with respect to the $\mathrm{Cu}$ substrate atoms. In Figure 2 (a, b upper panels) ball-and-stick models for both adsorption conformations on $\mathrm{Cu}(111)$ are represented in a top view for both 


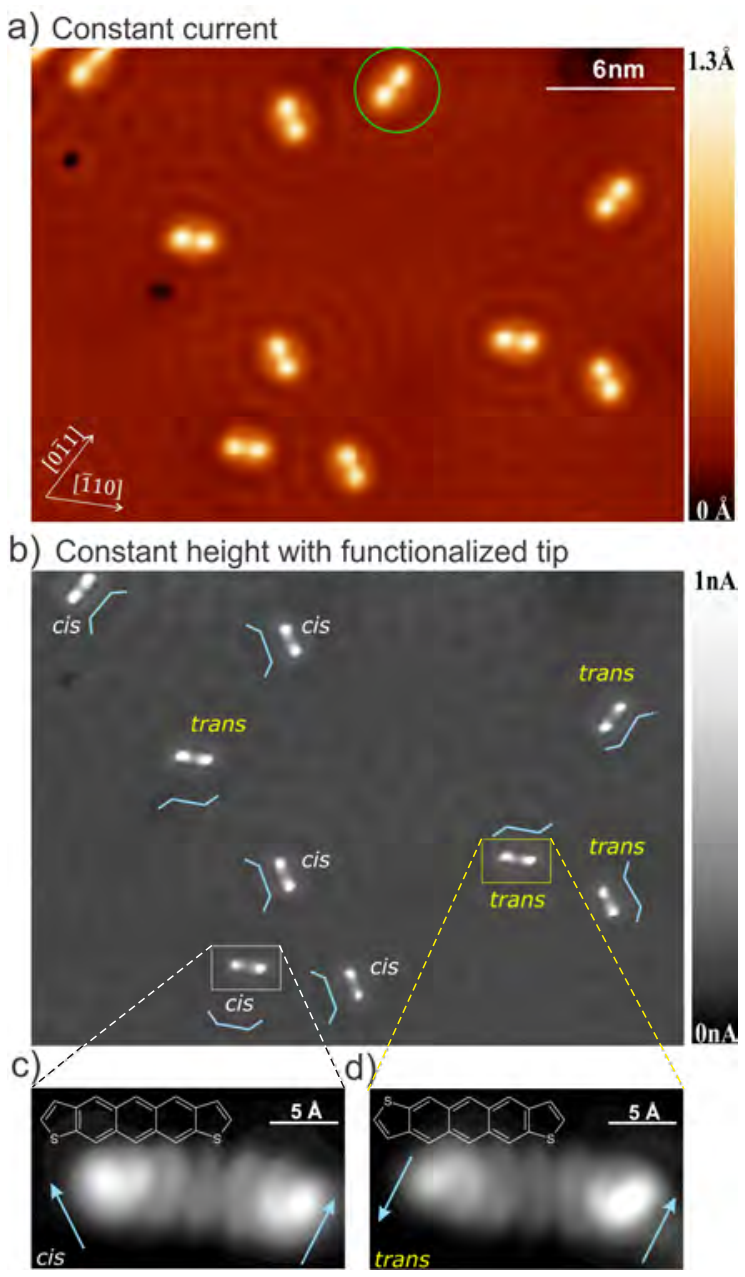

Figure 1: a) $30 \times 20 \mathrm{~nm}^{2}$ STM topography acquired in constant current mode ( $I=100 \mathrm{pA}$, $V=100 \mathrm{mV})$. ADT molecules are oriented along the high symmetry directions of the $\mathrm{Cu}(111)$ surface. The encircled molecule was used to functionalize the tip by vertical manipulation. b) Isomer-resolved STM image obtained with the functionalized tip of the same area as (a) in constant height $(\sim 5 \AA$ above the surface, $V=10 \mathrm{mV})$. Guiding lines are drawn for each molecular configuration. (c, d) Enlarged resolution images of the two ADT molecules framed in (b), represent the cis-ADT and trans-ADT isomers. Guiding arrows are drawn at the molecular extremities.

isomers. The acene group tends to have the benzene units centered over the hollow sites of the surface, which favors alignment of the molecules along the high symmetry directions as in the case of pentacene on $\mathrm{Cu}(111) \cdot{ }^{35}$ In addition, there is a tendency of the $\mathrm{S}$ atoms of the thiophene rings to sit on top of $\mathrm{Cu}$ atom positions. ${ }^{36}$ The cis-isomer has its acene rings centered over the hollow sites and follows the high symmetry axis with the S atoms in atop positions of the $\mathrm{Cu}(111)$ surface. Conversely, for the trans-isomer, when the acene rings 

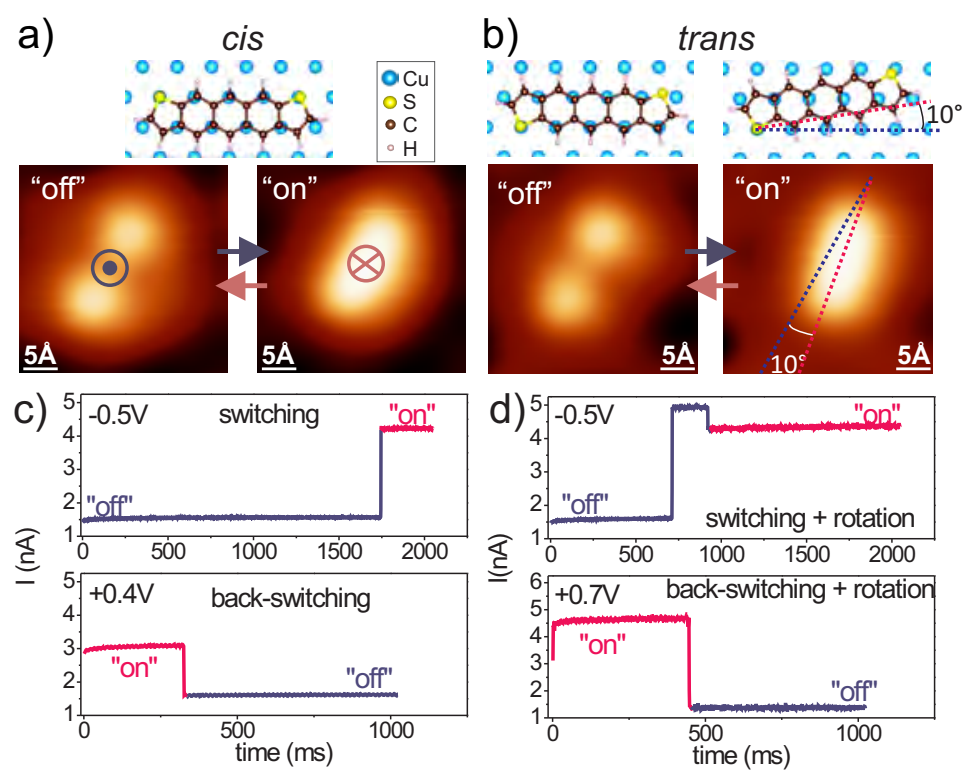

Figure 2: (a,b) STM images of the cis-ADT isomer and trans-ADT isomer and the top view of the ball-and-stick models representations on $\mathrm{Cu}(111)$, in the "off" and "on" conformation, respectively. Applying a negative bias pulse above the center of the molecules as indicated, implies switching to the "on" conformation. Trans-ADT isomers rotate by about $10^{\circ}$ during the switching process. (c,d) Current monitored as a function of time for the switching and back-switching process in an open-loop configuration. Before the feedback was tuned off the tip was stabilized at $100 \mathrm{mV}$ and $100 \mathrm{pA}$. Subsequently the tip was approached by $1 \AA$ (and $0.8 \AA$ for (d)-lower panel, respectively) towards the molecule's center with the feedbackloop turned off. Upper panel: switching process of cis-ADT and trans-ADT isomers from the "off" state (lower conductance) to the "on" state (higher conductance), by applying a negative bias voltage. Lower panel: back-switching from "on" to "off" conformation by applying a positive bias voltage.

are centered over the hollow sites the $\mathrm{S}$ atoms cannot be in the same registry with $\mathrm{Cu}(111)$ atoms of the surface. This suggest a reason for why the cis-isomers do not change the adsorption position while the trans-isomers rotate, as shown in panel (b). DFT calculations of the adsorption energy for both isomers and both conformations ("off" and "on" states) as a function of the tilt angle of the molecules with respect to the surface symmetry axis show minima at $0^{\circ}$. For the trans-isomer in the "on" state a small energy barrier for a rotation by about $10^{\circ}$, has to be overcome. (Supplementary Information)

The detailed electronic density of states of the molecular conformations is summarized in Figure 3. Using an STM tip functionalized with an ADT molecule, the orbital structures 

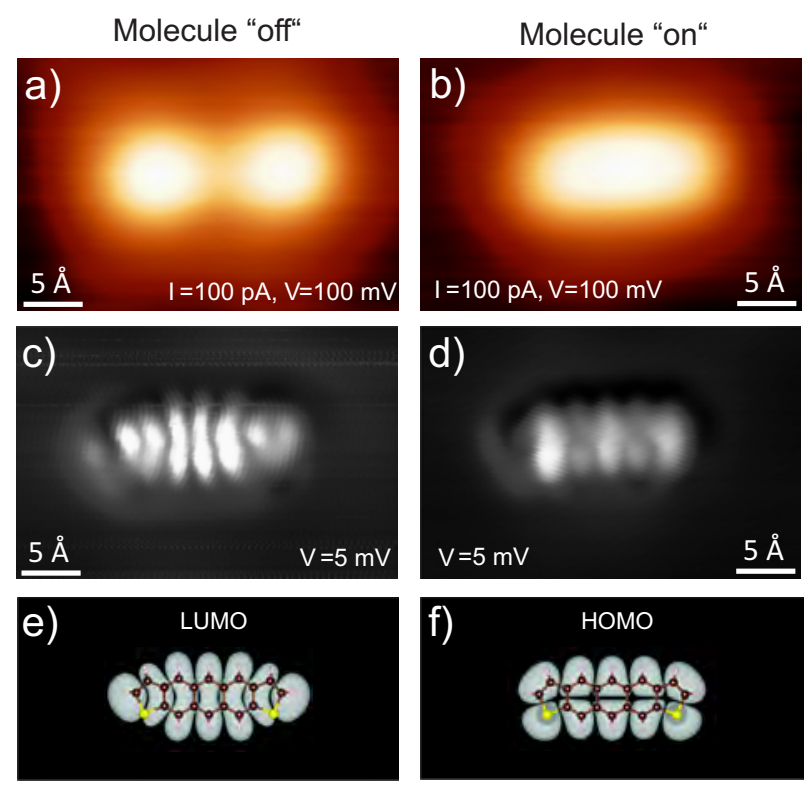

Figure 3: (a,b) STM topographic images of a cis-ADT molecule in "off" and "on" conformations, respectively. (c, d) Constant height images resolving the orbital structures of the "off" and "on" conformations, resembling the LUMO and HOMO of a free molecule in the gas phase as shown in the (e) and (f) panels.

of both conformations ("off" and "on") close to the Fermi level of the substrate (recorded at $5 \mathrm{mV}$ ) are resolved (Figure $3 \mathrm{c}, \mathrm{d}$ ). Comparing the images with the molecular orbitals calculated for the free molecule in the gas phase reveals that the states near the $E_{F}$ in the "off" state resemble the lowest unoccupied molecular orbital (LUMO), whereas in the "on" state they resemble the highest occupied molecular orbital (HOMO). This observation suggests that the switching between the two conformations is related to a charge transfer accompanied by a shift of the molecular orbitals. This behavior during the switching process, which is related to changes of the alignment of the energy levels, is obtained for both isomers. The only difference, as pointed out already, is the additional in-plane rotation in the case of the trans-ADT. Thus, the switching mechanism itself is similar for both isomers and for simplicity we will focus in the following on the cis-ADT.

To obtain a clear understanding of these two molecular conformations, DFT calculations of the electronic structure of the molecule-substrate system varying the adsorption distance, were performed. As described below, the bent adsorption geometry of the ADT in the "off" 

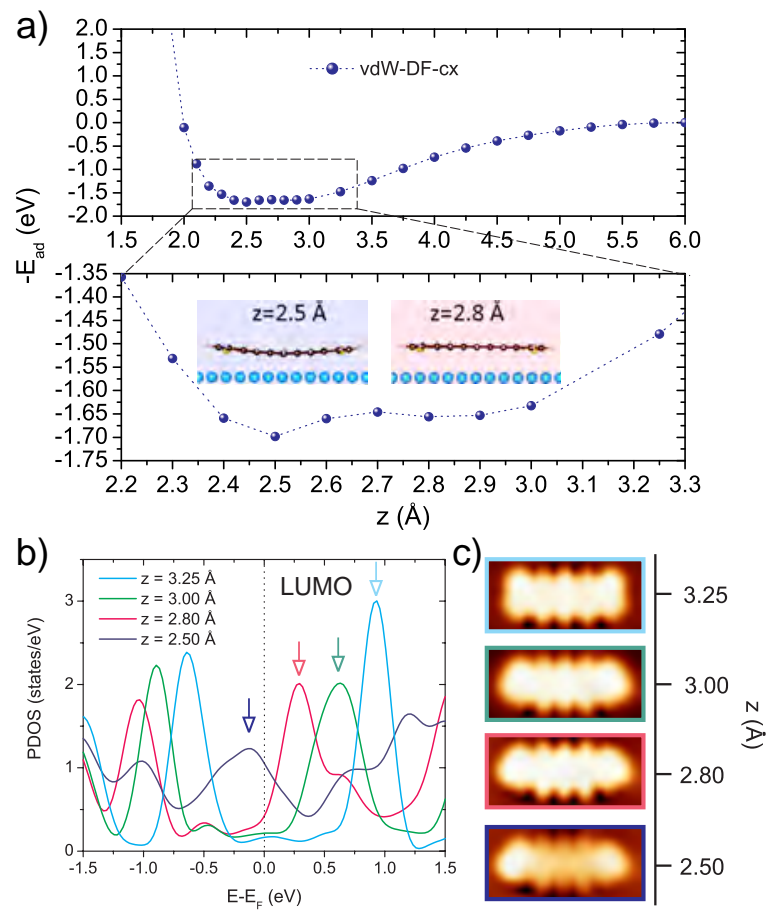

Figure 4: a) Adsorption energy $-E_{a d}$ of the cis-ADT isomer as a function of the adsorption height $z$ on $\mathrm{Cu}(111)$ from vdW-DF-cx calculations. Lower panel: close-up around the minima of the adsorption curve. Insets: side view of the optimized geometries of the ADT molecule at the two adsorption heights $z=2.5 \AA$ and $z=2.8 \AA$, where the two minima in $-E_{a d}$ appear, corresponding to the "off" and "on" conformations, respectively. b) The PDOS calculated at the molecular adsorption distance of $z=3.25 \AA, z=3 \AA, z=2.8 \AA$ and $z=2.5 \AA$ highlights shifts of the LUMO decreasing the distance to the surface, as indicated by arrows. A partial filling of the LUMO orbital is obtained for the molecule in the "off" conformation at $z=2.5 \AA$. c) Calculated molecular appearances at the corresponding distance above the surface. The geometry for adsorption distances of $z=3 \AA$ and $z=3.25 \AA$ are not fully optimized.

state permits a closer adsorption distance, as compared to the planar case in the "on" state, that leads to a rather strong hybridization between the ADT molecule's $\pi$-cloud and the $\mathrm{Cu}(111)$ metal surface, enough to compensate the Pauli repulsion and the energy penalty for the geometrical distortion. The calculations reveal two minima for the adsorption energies at $z=2.5 \AA$ and $z=2.8 \AA$ separated by a shallow energy barrier as shown in Figure 4 (a). These two minima appear after relaxing the $(\mathrm{X}, \mathrm{Y}, \mathrm{Z})$ coordinates of the $\mathrm{S}$ atoms and the (X,Y) coordinates of the $\mathrm{C}$ and $\mathrm{H}$ atoms. The corresponding fully optimized molecular conformations with full relaxations of all atoms in $(\mathrm{X}, \mathrm{Y}, \mathrm{Z})$ coordinates are indicated in the 
inset of Figure 4(a).

In the molecular state at $z=2.5 \AA$, the "off" conformation, the molecule is adsorbed with the acene rings slightly closer to the surface compared to the $\mathrm{S}-\mathrm{Cu}$ distance, which gives rise to the "dumbbell-like" appearance in the STM topographic images. This geometry corresponds to an adsorption state with a strong hybridization between the ADT molecule and the $\mathrm{Cu}(111)$ substrate that includes a significant amount of charge transfer to the LUMO of about one electron. By these means, the LUMO broadens and shifts to lower energies, thus closer to the Fermi energy, as visible in the projected density of states (PDOS) in Figure 4 (b). The "off" state is the ground state of the molecule, consistent with the experimental observation where molecules are only found in the "off" state after deposition.

In the "on" state the interaction between the ADT molecule and the $\mathrm{Cu}(111)$ substrate is weaker and corresponds to an adsorption state with a planar molecular geometry at larger distance of the acene rings from the surface. Here, no significant charge transfer between the surface and the molecule is present and the LUMO is essentially unoccupied as shown in the calculated PDOS (Figure $4 \mathrm{~b}$ ). By increasing the distance between the molecule and the surface, the LUMO shifts further towards higher energies. In both, the "on" and the "off" state, a hybridization between the ADT molecular orbitals and $\mathrm{Cu}(111)$ surface states exists. However, the dependence of the hybridization and the mixing of the HOMO-LUMO orbitals decreases by increasing the molecular adsorption distance. These theoretical findings support the experimental observations. In the "off" state the LUMO shifts down to the Fermi energy, whereas in the "on" state, the LUMO is shifted to higher energies. The exact energy positions of the HOMO and LUMO molecular orbitals with respect to the metal states is difficult to accurately describe by DFT. The calculations still show a contribution of the LUMO at the Fermi level of the substrate even for an adsorption distance of $z=2.8 \AA$ (Figure $4 \mathrm{c}$ ). However, the shift of the LUMO to higher energies with the adsorption distance is well described, supporting the experimental findings. A schematic representation of the alignment of the energy levels, based on the calculated PDOS, in the two conformations and for both 
switching processes is depicted in Figure 5 (a,b).

In the "off" state, the ADT molecule is in a bent adsorption geometry with the acene rings close to the $\mathrm{Cu}(111)$ surface and the LUMO orbital partially occupied. By applying a negative bias voltage pulse the LUMO is depopulated destabilizing electronically the ADT, which facilitates the switching, i.e., the switch to the "on" state with a planar geometry at larger distances. The reverse process, at positive bias voltage, is accompanied by a charge transfer into the LUMO, leading to the back-switching to the "off" state.

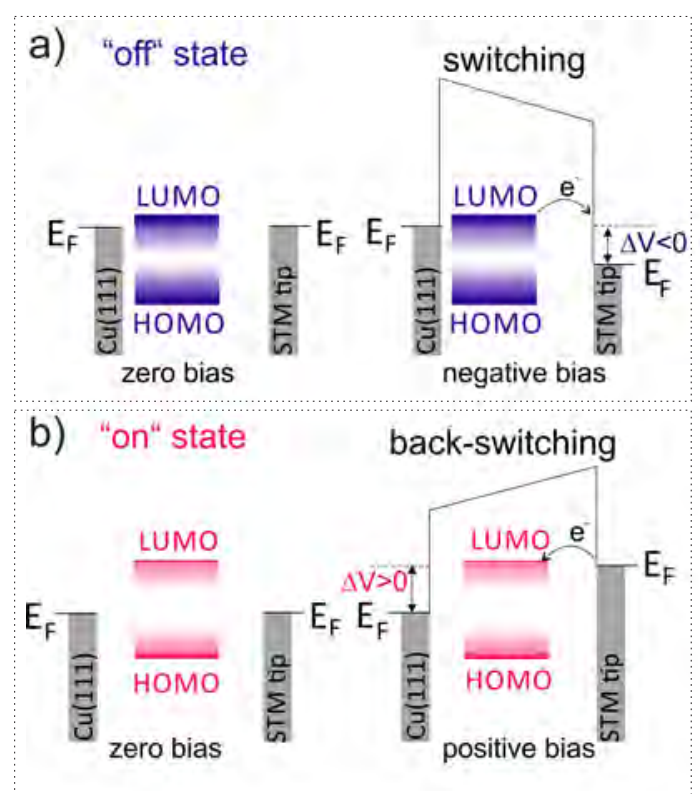

Figure 5: (a,b) Schematic representation of the energy level alignment for the molecule in both conformations showing a depopulation and population of the LUMO upon switching and back-switching, respectively.

The switching and back-switching processes are induced at opposite polarities of the bias voltage, showing a characteristic hysteresis of the tunneling current while ramping the bias voltage, i.e. it reflects a standard behavior of bipolar conductance switches (Figure 6 a). The two step-like features in the current-voltage characteristic correspond to the switching and back-switching process. The threshold voltage and threshold current of the switching and back-switching processes are determined by measuring several hysteresis curves on top of different molecules and at different tip-sample distances and different sweeping times (from $10 \mathrm{~s}$ to maximum $50 \mathrm{~s}$ ). In Figure 6 (b), the histogram of the bias voltages with both 
polarities is plotted, corresponding to the "on" state and to the "off" state switching. In Figure 6 (c), the distribution of the current values for both switching processes is represented. A broader range of voltage values are observed for the switching events at negative polarity, while for the back-switching at positive bias polarity, the distribution of current values is broader. This slightly different distribution range of voltage and current for the switching and back-switching processes are related to different switching mechanisms attributed to the depopulation and population of the LUMO orbital. The bipolar switching process, where switching takes place at negative energies and back-switching at positive ones, is confined within an energy range of $\pm 0.6 \mathrm{~V}$ (for moderate tunnel parameters, i.e. currents no larger than approximately $20 \mathrm{nA}$ ). Beyond that energy window, for instance at $-0.8 \mathrm{~V}$, current fluctuations associated with a toggling between the two states are observed. However, the switching mechanisms involved at those energies differ from those responsible for the bipolar switching. The current fluctuations show that the "off" state is energetically more favorable as the occupation time of the molecule in the "off" state is much higher compared to the occupation time of the molecule in the "on" state (Supplementary Information).

To confirm that the "on" conformation is the metastable state we have tracked the duration of the "on" state of the cis molecules over time. Figure 7 shows the percentage of the cis molecules in the "on" state which decreases exponentially in time with a decay time of about 7 hours.

The switching and back-switching processes correspond to electronic transitions accompanied by conformational changes between two different potential energy surfaces. These energy surfaces are defined by the Marcus parabolas of the corresponding diabatic states, ${ }^{37}$ similar to the case of metal atoms adsorbed on thin $\mathrm{NaCl}$ buffer layer grown on metal surfaces. ${ }^{38}$ However, in our case the conformational changes occur at the molecular level of individual ADT molecules, which possess many degrees of freedom and, thus, allow for the existence of the two above mentioned adsorption states: a bent geometry with the acene rings at closer distances, in which the optimal $\pi$-bonding length of the central acene rings 
a)

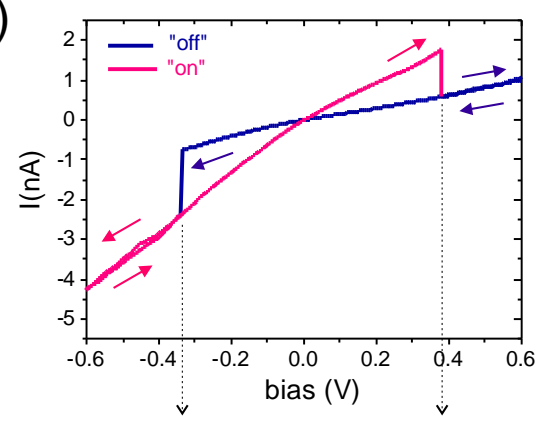

b) switching back-switching
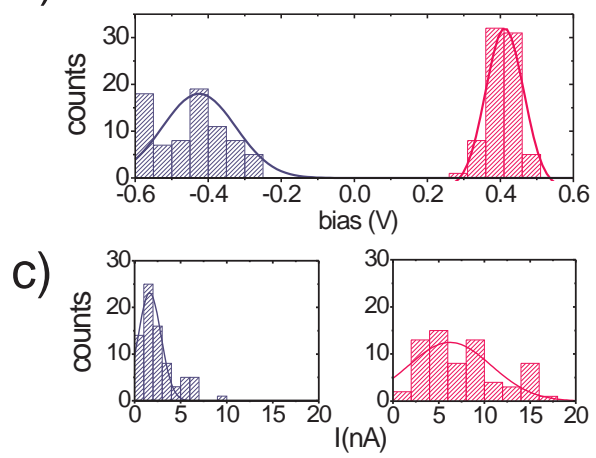

Figure 6: a) Bipolar switching and back-switching hysteresis recorded by positioning the STM tip over the center of a cis-ADT isomer in the "off" conformation. The $I(V)$ curves were recorded as the bias voltage was ramped, with the feedback turned off, from $0.6 \mathrm{~V}$ to $-0.6 \mathrm{~V}$ and back as indicated by arrows in corresponding colors. The two step-like features in the current traces correspond to the switching and back-switching process. b) Bias voltage histograms with a bin of $50 \mathrm{mV}$ corresponding to the switching (mean value $-0.44 \pm 0.1 \mathrm{~V}$ ) and back-switching (mean value $+0.4 \pm 0.04 \mathrm{~V}$ ) processes, evaluated from several hysteresis curves taken above different molecules, at different tip-sample distances and with different sweeping times (from $10 \mathrm{~s}$ to maximum $50 \mathrm{~s}$ ). c) Current histograms of the switching (mean value $2.52 \pm 1.88 \mathrm{nA}$ ) and back-switching (mean value $6.06 \pm 3.68 \mathrm{nA}$ ) processes.

compensates for the energy cost of the distortion imposed by the constraint of the S-Cu bond distance of the S-containing end groups, and a planar geometry at larger distances of the acene rings above the surface and optimal S-Cu bond distances. The optimal adsorption distance between the central acene groups and the $\mathrm{Cu}(111)$ surface is very close to the pentacene- $\mathrm{Cu}(111)$ adsorption distance. ${ }^{33,34}$ However, the presence of $\mathrm{S}$ atoms in both end groups of the ADT molecule imposes an additional constraint due to the longer S-Cu bonding distance. The combination of both effects results in the two adsorption geometries, a bent geometry ("off" state) in which energy costs of the deformation is compensated by the 
a)

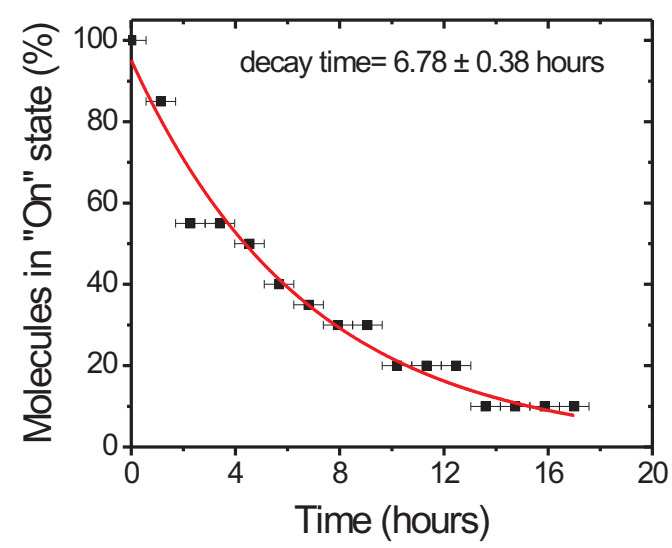

b)

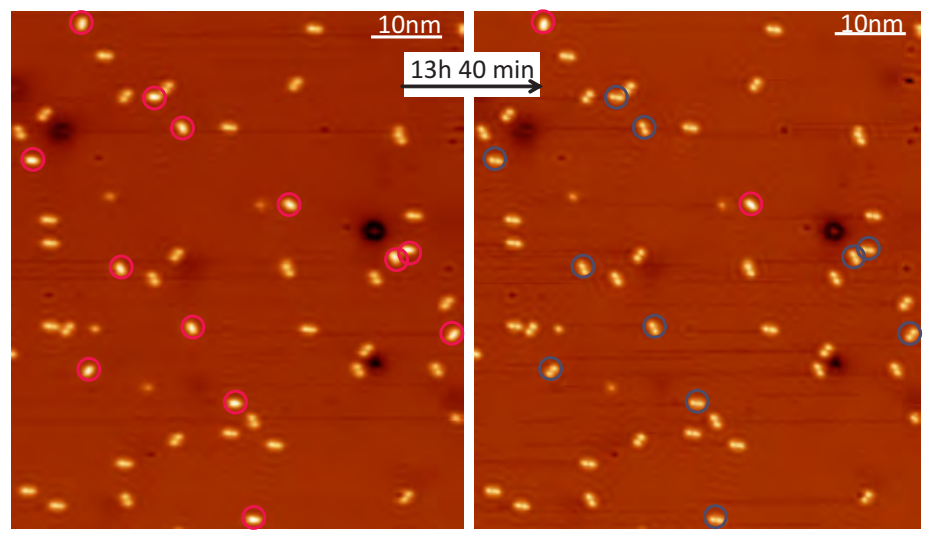

Figure 7: a) Exponential decay time of the molecules from the "on" to the "off" state. b) The corresponding STM images acquired in constant current mode $(I=40 \mathrm{pA}, V=50 \mathrm{mV})$ with several molecules in "on" state and after they are switched to the "off" state.

optimal $\pi$-bonding of the acene groups to the metal surface, and a planar geometry ("on" state) without energy penalty for the geometrical distortion but with a longer bond length due to the $\mathrm{S}-\mathrm{Cu}$ constraint. A similar behavior is described in the case of chlorinated benzene derivatives, as discussed in Ref. [29], but not in the case of pentacene. In this latter case there are no additional atoms, like the $\mathrm{S}$ atoms of $\mathrm{ADT}$ or the $\mathrm{Cl}$ atoms of benzene derivatives, introducing constraints to the optimal adsorption geometries. Therefore, it is the molecular flexibility which allows the molecule to possess the two different bonding conformations to the metal surface, accompanied by changes of the molecular orbital occupations.

\section{Conclusion}

In conclusion, we have demonstrated reversible bipolar conductance switching of single ADT molecules adsorbed on $\mathrm{Cu}(111)$ and induced locally in a fully controlled manner in the STM tunneling junction. The comparison of the orbital resolved STM images with DFT calculations reveals that the switching process is accompanied by a geometric and an electronic change induced by charge transfer at the metal-organic interface associated with the depopulation and population of the LUMO orbital. These findings may be transferred to potential 
storage bits, allowing for controlled switching to "on" and "off" states at the individual molecular level.

\section{Experimental and computational methods}

The experiments were performed under ultra-high vacuum conditions (UHV) with a homebuilt low temperature Scanning Tunneling Microscope (LT-STM) operating at a temperature of $6 \mathrm{~K}$. The single $\mathrm{Cu}(111)$ crystal was prepared by repeated cycles of $\mathrm{Ar}^{+}$ion sputtering and subsequent annealing. A mixture of cis:anthra[2,3-b:7,6- $\left.b^{\prime}\right]$ dithiophene and trans:anthra[2,3$\left.b: 6,7-b^{\prime}\right]$ dithiophene isomers were sublimated with an Organic Molecular Beam Deposition technique on the $\mathrm{Cu}(111)$ surface with a submonolayer coverage. ADT molecules were purchased from Sigma-Aldrich. To prevent the molecules from reacting at the surface, the substrate is held at $200 \mathrm{~K}$ during deposition. Subsequently, the sample is transferred in situ to the LT-STM. Bias voltages are applied to the sample. The STM measurements were performed both in constant current and constant height modes.

The DFT-vdW calculations were done using the VASP code. ${ }^{39,40}$ The ADT/Cu(111) system was modelled using a $8 \times 4 \mathrm{Cu}(111)$ periodic supercell with four $\mathrm{Cu}$ layers, the ionelectron interaction was described with the Projector Augmented-Wave (PAW) method, ${ }^{41}$ whereas the exchange and correlation potential was taken into account by the Generalized Gradient Approximation(GGA) ${ }^{42}$ and including the vdW dispersion forces using the vdWDF-cx method. ${ }^{31,32}$ In the plane wave expansion we consider a kinetic energy cut-off of $500 \mathrm{eV}$. To satisfy the summations in the reciprocal space for the Brillouin zone a mesh of $2 \times 3 \mathrm{k}$ points in the $1 \times 1$ unit cell was chosen. For all calculations the electronic convergence

criterion was $1 \times 10^{-4} \mathrm{eV}$, while the convergence on forces in the relaxation was $0.05 \mathrm{eV} / \AA$. 


\section{Acknowledgement}

We acknowledge funding by the Emmy-Noether-Program of the Deutsche Forschungsgemeinschaft, the SFB 767, and the Baden-Württemberg Stiftung. R.P. and A.A. thank the basque Departamento de Universidades e Investigacion (grant No. IT-756-13) and the spanish Ministerio de Economia y Competitividad (grant No. FIS2013-48286-C2-8752-P) for financial support. R.P. and A.A. also acknowledge Joakim Löfgren and Per Hyldgaard for stimulating discussions about the vdW-DF-cx implementation.

\section{Supporting Information Available}

This material is available free of charge via the Internet at http://pubs.acs.org/.

\section{References}

1. Van der Molen, S. J.; Naaman, R.; Scheer, E.; Neaton, J. B.; Nitzan, A.; Natelson, D.; Tao, N.; Van der Zant, H.; Mayor, M.; Ruben, M. et al. Visions for a Molecular Future. Nat. Nanotechnol. 2013, 8, 385-389.

2. Kay, E. R.; Leigh, D. A.; Zerbetto, F. Synthetic Molecular Motors and Mechanical Machines. Angew. Chem., Int. Ed. 2007, 46, 72-191.

3. Feringa, B. L.; Browne, W. R. Molecular Switches (2nd Edition); Wiley-VCH: WEINHEIM, 2011; pp 1-792.

4. Eigler, D. M.; Schweizer, E. K. Positioning Single Atoms with a Scanning Tunnelling Microscope. Nature 1990, 344, 524-526.

5. Iancu, V.; Hla, S.-W. Realization of a Four-Step Molecular Switch in Scanning Tunneling Microscope Manipulation of Single Chlorophyll Molecules. Proc. Natl. Acad. Sci. 2006, 103, 13718-13721. 
6. Wang, Y.; Kröger, J.; Berndt, R.; Hofer, W. A. Pushing and Pulling a Sn Ion through an Adsorbed Phthalocyanine Molecule. J. Am. Chem. Soc. 2009, 131, 3639-3643.

7. Fu, Y.-S.; Schwöbel, J.; Hla, S.-W.; Dilullo, A.; Hoffmann, G.; Klyatskaya, S.; Ruben, M.; Wiesendanger, R. Reversible Chiral Switching of Bis(phthalocyaninato) Terbium(III) on a Metal Surface. Nano Lett. 2012, 12, 3931-3935.

8. Ohmann, R.; Vitali, L.; Kern, K. Actuated Transitory Metal - Ligand Bond As Tunable Electromechanical Switch. Nano Lett. 2010, 10, 2995-3000.

9. Albrecht, F.; Neu, M.; Quest, C.; Swart, I.; Repp, J. Formation and Characterization of a Molecule-Metal-Molecule Bridge in Real Space. J. Am. Chem. Soc. 2013, 135, 9200-9203.

10. Mohn, F.; Repp, J.; Gross, L.; Meyer, G.; Dyer, M. S.; Persson, M. Reversible Bond Formation in a Gold-Atom-Organic-Molecule Complex as a Molecular Switch. Phys. Rev. Lett. 2010, 105, 266102.

11. Leung, L.; Lim, T.; Polanyi, J. C.; Hofer, W. A. Molecular Calipers Control Atomic Separation at a Metal Surface. Nano Lett. 2011, 11, 4113-4117.

12. Swart, I.; Sonnleitner, T.; Repp, J. Charge State Control of Molecules Reveals Modification of the Tunneling Barrier with Intramolecular Contrast. Nano Lett. 2011, 11, $1580-1584$.

13. Walch, H.; Leoni, T.; Guillermet, O.; Langlais, V.; Scheuermann, A.; Bonvoisin, J.; Gauthier, S. Electromechanical Switching Behavior of Individual Molecular Complexes of $\mathrm{Cu}$ and $\mathrm{Ni}$ on NaCl-Covered $\mathrm{Cu}(111)$ and $\mathrm{Ag}(111)$. Phys. Rev. B 2012, 86, 075423.

14. Fu, Y.-S.; Zhang, T.; Ji, S.-H.; Chen, X.; Ma, X.-C.; Jia, J.-F.; Xue, Q.-K. Identifying Charge States of Molecules with Spin-Flip Spectroscopy. Phys. Rev. Lett. 2009, 103, 257202 . 
15. Leoni, T.; Guillermet, O.; Walch, H.; Langlais, V.; Scheuermann, A.; Bonvoisin, J.; Gauthier, S. Controlling the Charge State of a Single Redox Molecular Switch. Phys. Rev. Lett. 2011, 106, 216103.

16. Uhlmann, C.; Swart, I.; Repp, J. Controlling the Orbital Sequence in Individual CuPhthalocyanine Molecules. Nano Lett. 2013, 13, 777-780.

17. Riedel, D.; Bocquet, M.-L.; Lesnard, H.; Lastapis, M.; Lorente, N.; Sonnet, P.; Dujardin, G. Selective Scanning Tunnelling Microscope Electron-Induced Reactions of Single Biphenyl Molecules on a Si(100) Surface. J. Am. Chem. Soc. 2009, 131, 7344-7352.

18. Liljeroth, P.; Repp, J.; Meyer, G. Current-Induced Hydrogen Tautomerization and Conductance Switching of Naphthalocyanine Molecules. Science 2007, 317, 1203-1206.

19. Henzl, J.; Mehlhorn, M.; Gawronski, H.; Rieder, K.-H.; Morgenstern, K. Reversible CisTrans Isomerization of a Single Azobenzene Molecule. Angew. Chem., Int. Ed. 2006, 45, 603-606.

20. Morgenstern, K. Isomerization Reactions on Single Adsorbed Molecules. Acc. Chem. Res. 2009, 42, 213-223.

21. Kumagai, T.; Hanke, F.; Gawinkowski, S.; Sharp, J.; Kotsis, K.; Waluk, J.; Persson, M.; Grill, L. Thermally and Vibrationally Induced Tautomerization of Single Porphycene Molecules on a $\mathrm{Cu}(110)$ Surface. Phys. Rev. Lett. 2013, 111, 246101.

22. Simpson, G. J.; Hogan, S. W. L.; Caffio, M.; Adams, C. J.; Früchtl, H.; van Mourik, T.; Schaub, R. New Class of Metal Bound Molecular Switches Involving H-Tautomerism. Nano Lett. 2014, 14, 634-639.

23. Parschau, M.; Passerone, D.; Rieder, K.-H.; Hug, H. J.; Ernst, K.-H. Switching the Chirality of Single Adsorbate Complexes. Angew. Chem., Int. Ed. 2009, 48, 4065-4068. 
24. Alemani, M.; Peters, M. V.; Hecht, S.; Rieder, K.-H.; Moresco, F.; Grill, L. Electric Field-Induced Isomerization of Azobenzene by STM. J. Am. Chem. Soc. 2006, 128, $14446-14447$.

25. Serrate, D.; Moro-Lagares, M.; Piantek, M.; Pascual, J. I.; Ibarra, M. R. Enhanced Hydrogen Dissociation by Individual Co Atoms Supported on $\operatorname{Ag}(111)$. J. Phys. Chem. C 2014, 118, 5827-5832.

26. Qiu, X. H.; Nazin, G. V.; Ho, W. Mechanisms of Reversible Conformational Transitions in a Single Molecule. Phys. Rev. Lett. 2004, 93, 196806.

27. Wu, S. W.; Ogawa, N.; Ho, W. Atomic-Scale Coupling of Photons to Single-Molecule Junctions. Science 2006, 312, 1362-1365.

28. Lee, J.; Tallarida, N.; Rios, L.; Perdue, S. M.; Apkarian, V. A. Single Electron Bipolar Conductance Switch Driven by the Molecular Aharonov-Bohm Effect. ACS Nano 2014, 8, 6382-6389.

29. Liu, W.; Filimonov, S. N.; Carrasco, J.; Tkatchenko, A. Molecular Switches from Benzene Derivatives Adsorbed on Metal Surfaces. Nat. Commun. 2013, 4, 3569.

30. Anthony, J. E. Functionalized Acenes and Heteroacenes for Organic Electronics. Chem. Rev. 2006, 106, 5028-5048.

31. Berland, K.; Hylgaard, P. Exchange Functional that Tests the Robustness of the Plasmon Description of the Van der Waals Density Functionals. Phys. Rev. B 2014, 89, 035412-1, $035412-8$.

32. Björkman, T. Testing Several Recent van der Waals Density Functionals for Layered Structures. J. Chem. Phys. 2014, 141, 074708-1, 074708-6. 
33. Toyoda, K.; Nakanoa, Y.; Hamada, I.; Lee, K.; Yanagisawa, S.; Morikawa, Y. FirstPrinciples Study of the Pentacene/Cu(1 11 1) Interface: Adsorption States and Vacuum Level Shifts. J. Electron Spectrosc. Relat. Phenom. 2009, 174, 78-84.

34. Koch, N.; Gerlach, A.; Duhm, S.; Glowatzki, H.; Heimel, G.; Vollmer, A.; Sakamoto, Y.; Suzuki, T.; Zegenhagen, J.; Rabe, J. P. et al. Adsorption-Induced Intramolecular Dipole: Correlating Molecular Conformation and Interface Electronic Structure. J. Am. Chem. Soc. 2008, 130, 7300-7304.

35. Lagoute, J.; Kanisawa, K.; Fölsch, S. Manipulation and Adsorption-Site Mapping of Single Pentacene Molecules on $\mathrm{Cu}(111)$. Phys. Rev. B 2004, 70, 245415.

36. Milligan, P.; Namarra, J. M.; Murphy, B.; Cowie, B.; Lennon, D.; Kadodwala, M. A NIXSW and NEXAFS Investigation of Thiophene on Cu(111). Surf. Sci. 1998, 412413, $166-173$.

37. Marcus, R. A. Electron Transfer Reactions in Chemistry. Theory and Experiment. Rev. Mod. Phys. 1993, 65, 599-610.

38. Olsson, F. E.; Paavilainen, S.; Persson, M.; Repp, J.; Meyer, G. Multiple Charge States of Ag Atoms on Ultrathin NaCl Films. Phys. Rev. Lett. 2007, 98, 176803.

39. Kresse, G.; Hafner, J. Ab Initio Molecular-Dynamics Simulation of the Liquid-MetalAmorphous-Semiconductor Transition in Germanium. Phys. Rev. B 1994, 49, 1425114269.

40. Kresse, G.; Furthmuller, J. Efficient Iterative Schemes for Ab Initio Total-Energy Calculations Using a Plane-Wave Basis Set. Phys. Rev. B 1996, 54, 11169-1118.

41. Blöchl, P. E. Projector Augmented-Wave Method. Phys. Rev. B 1994, 50, 17953-17979.

42. Perdew, J. P.; Burke, K.; Ernzerhof, M. Generalized Gradient Approximation Made Simple [Phys. Rev. Lett. 77, 3865 (1996)]. Phys. Rev. Lett. 1997, 78, 1396-1396. 
Graphical TOC Entry

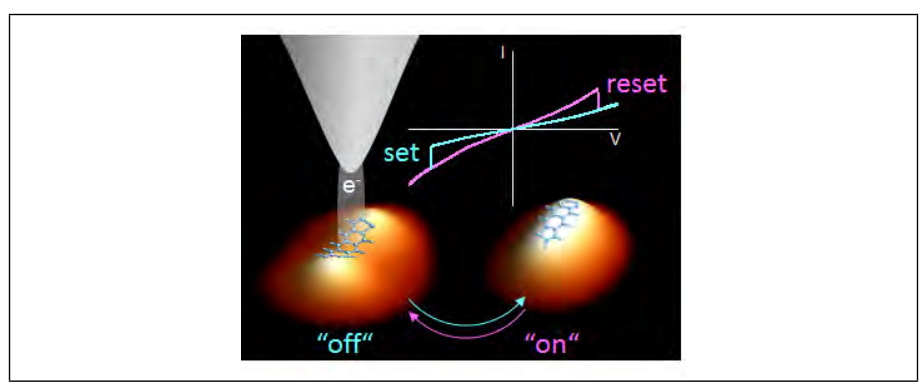




\section{Supplementary Information}

\section{Bipolar Conductance Switching of Single Anthradithiophene Molecules}

Bogdana Borca, ${ }^{*}{ }^{\dagger, \perp}$ Verena Schendel, ${ }^{\dagger} \perp$ Rémi Pétuya,${ }^{\dagger}$ Ivan Pentegov, ${ }^{\dagger}$ Tomasz Michnowicz, ${ }^{\dagger}$ Ulrike Kraft, ${ }^{\dagger}$ Hagen Klauk, ${ }^{\dagger}$ Andrés Arnau, ${ }^{\emptyset, \ddagger}$ Peter Wahl, ${ }^{\dagger, \S}$ Uta Schlickum, ${ }^{*}{ }^{\dagger}$ and Klaus Kern ${ }^{\dagger}, \|$

\section{Analysis of switching behavior in time traces}

Anthradithiophene (ADT) molecular switches are interconverted between the bistable states corresponding to different adsorption conformations on the surface by a bias voltage with opposite polarities applied locally on individual molecules underneath the STM tip. The corresponding $I(V)$ curves show characteristic hysteretic behavior. Predominantly the switching from the "off" to the "on" state occurs at negative voltage polarity applied to the sample and the back-switching at positive polarity. For the negative polarity of the applied voltage with absolute values higher than $-0.6 \mathrm{~V}$, both transitions may occur. For positive polarity of the applied voltage, a current higher than $20 \mathrm{nA}$ cause the appearance of switching and back-switching transitions. At these parameters of instability, the current traces show step-like transitions corresponding to the switching and the back-switching processes, the so-called telegraphic noise behavior. In Figure S1 a), we represent two current traces recorded at $-0.8 \mathrm{~V}$ and $+0.4 \mathrm{~V}$ respectively, for which these transitions between the "off" and "on" states arise. The spectra reveal a much longer lifetime of the system in the "off" state as compared to the lifetime in the "on" state as revealed by the current histograms Figure S1 (b), which is consistent with the fact that the "off" state is found as the ground state and the "on" state as a metastable state, in agreement with our DFT calculations.

\footnotetext{
*To whom correspondence should be addressed, ${ }^{\perp}$ Equal contribution

${ }^{\dagger}$ Max-Planck-Institute for Solid State Research, 70569 Stuttgart, Germany

Donostia International Physics Centre, E-20018 Donostia - San Sebastián, Spain

"Departamento de Fisica de Materiales UPV/EHU and CFM Donostia, Centro Mixto CSICUPV/EHU, E-20018 Donostia - San Sebastián, Spain

${ }^{\S}$ SUPA, School of Physics and Astronomy, University of St Andrews, North Haugh, St Andrews, KY16 9SS, United Kingdom

"Institut de Physique de la Matière Condensée, École Polytechnique Fédérale de Lausanne (EPFL), CH-1015 Lausanne, Switzerland
} 

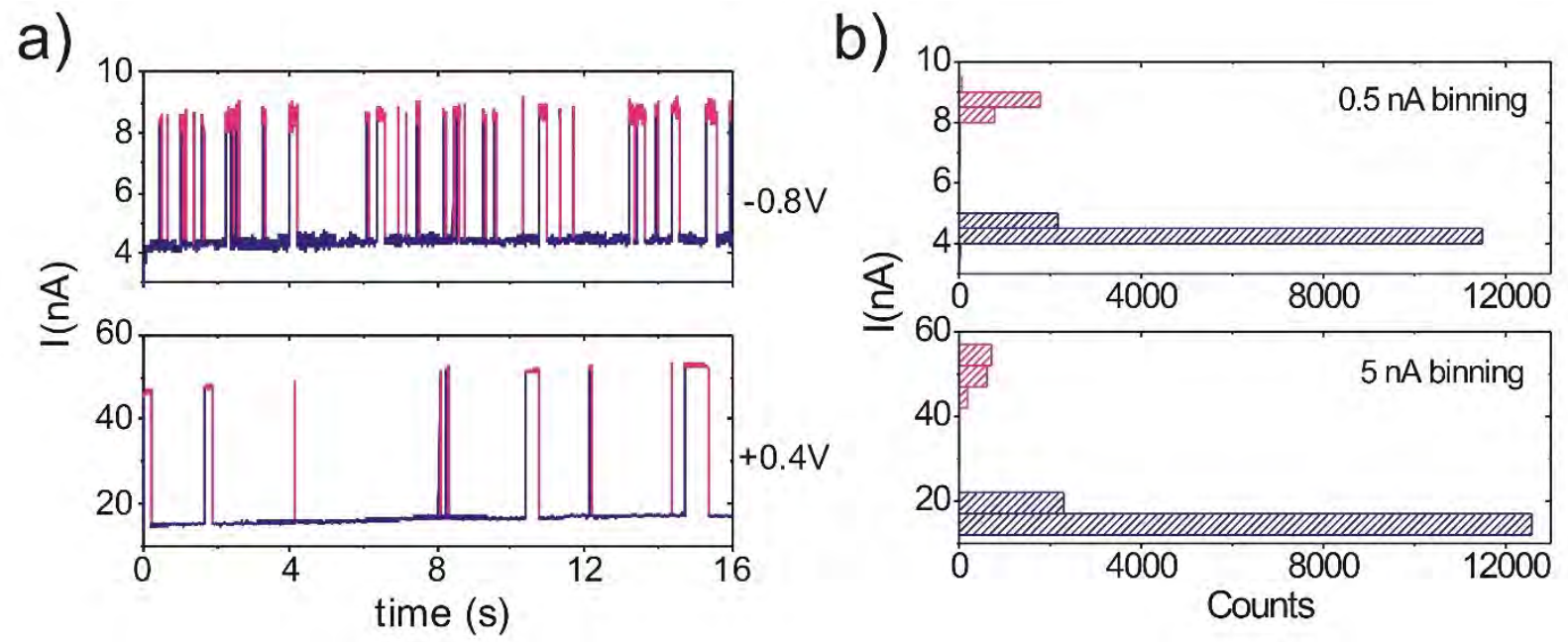

Figure S1 a) Current traces recorded at $-0.8 \mathrm{~V}$ and $+0.4 \mathrm{~V}$ with open feedback loop. b) Histograms of the current traces in panel (a).

\section{Adsorption energy as function of tilt angle}

The adsorption conformations of ADT molecules rely on the interplay between the sulfur containing groups and the acene group position with the substrate atoms. Figure S2 summarizes the analysis of the adsorption energy for both isomers and both conformations at $z=2.50 \mathrm{~A}$ ("off" state) and $z=2.80 \AA$ ("on" state) as a function of the tilt angle of the molecules with respect to the surface symmetry axis. The acene group tends to have the benzene units centered over the hollow sites of the surface, which favors the alignment of the molecules along the high symmetry directions, particularly at short distances to the surface when the ADT molecules are in the "off" state and in a stronger interaction with the surface. In these conditions the adsorption curves show clear minima at $0^{\circ}$ tilt angle for both isomers (Figure S2 a). However, there is an additional effect due to the tendency of the sulfur atoms of the thiophene rings to adopt atop positions that reinforces the alignment for the cis-isomer but tends to rotate the ADT in the case of the transisomer, the balance being very delicate as shown in the right panel of Figure S2 (a). A ball-andstick model of both isomers is drawn in Figure $\mathrm{S} 2(\mathrm{~b}, \mathrm{c})$ for $0^{\circ}$ and $9^{\circ}$ tilt angle, respectively .

As a consequence of these two competing effects, in the "on" state, the cis-isomer has an energy barrier of $200 \mathrm{meV}$ for the rotation higher by a factor of four than that of the trans-isomer which is only $50 \mathrm{meV}$. These findings explain why only the trans-isomer rotates during the switching process. However, such a delicate balance is not fully reproduced by our DFT calculations with a steepest minimum at $0^{\circ}$ tilt angle instead of $9^{\circ}$ for the trans-isomer at $z=2.80 \AA$ (in the "on" state). 
a)

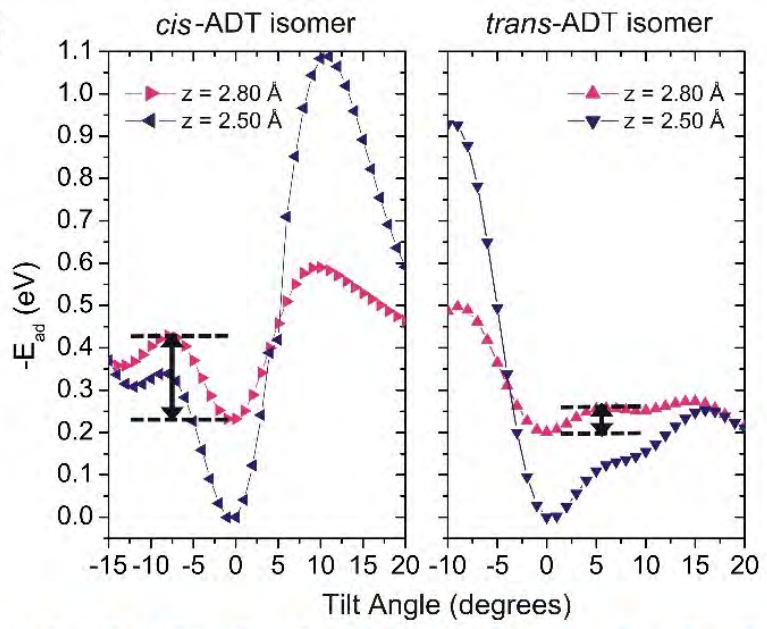

b)

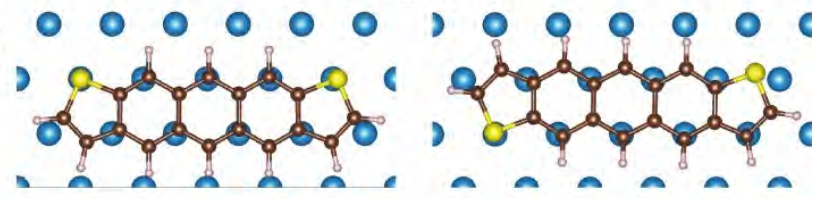

c)

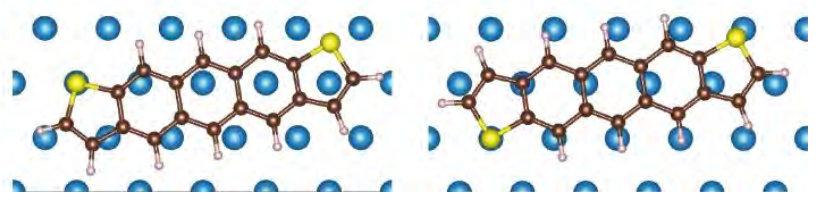

Figure S2 a) Adsorption energy $-E_{a d}$ of the cis-ADT and trans-ADT isomers as a function of the tilt angle in the two adsorption states: "off" state at $z=2.50 \AA$ and "on" state at $z=2.80 \AA$ A. The arrows mark the energy barriers for the rotation that are $200 / 50 \mathrm{meV}$ for the cis-/transisomer, respectively. $(\mathrm{b}, \mathrm{c})$ Top view of the cis-ADT (left panels) and trans-ADT (right panels) in the equilibrium position at $z=2.80 \AA$ A following the high symmetry crystallographic axes of $\mathrm{Cu}(111)$ in (b) and tilted by an angle of $9^{\circ}$ around the sulfur atom on top position on the left of the molecule in (c). 